\title{
Factors associated with suicidal thoughts in a large community study of older adults
}

Osvaldo P. Almeida, Brian Draper, John Snowdon, Nicola T. Lautenschlager, Jane Pirkis, Gerard Byrne, Moira Sim, Nigel Stocks, Leon Flicker and Jon J. Pfaff

\section{Background}

Thoughts about death and self-harm in old age have been commonly associated with the presence of depression, but other risk factors may also be important.

\section{Aims}

To determine the independent association between suicidal ideation in later life and demographic, lifestyle, socioeconomic, psychiatric and medical factors.

\section{Method}

A cross-sectional study was conducted of a communityderived sample of 21290 adults aged 60-101 years enrolled from Australian primary care practices. We considered that participants endorsing any of the four items of the Depressive Symptom Inventory - Suicidality Subscale were experiencing suicidal thoughts. We used standard procedures to collect demographic, lifestyle, psychosocial and clinical data. Anxiety and depressive symptoms were assessed with the Hospital Anxiety and Depression Scale.

\section{Results}

The 2-week prevalence of suicidal ideation was 4.8\%. Male gender, higher education, current smoking, living alone, poor social support, no religious practice, financial strain, childhood physical abuse, history of suicide in the family, past depression, current anxiety, depression or comorbid anxiety and depression, past suicide attempt, pain, poor selfperceived health and current use of antidepressants were independently associated with suicidal ideation. Poor social support was associated with a population attributable fraction of $38.0 \%$, followed by history of depression $(23.6 \%)$, concurrent anxiety and depression (19.7\%), prevalent anxiety (15.1\%), pain (13.7\%) and no religious practice (11.4\%).

\section{Conclusions}

Prevalent and past mood disorders seem to be valid targets for indicated interventions designed to reduce suicidal thoughts and behaviour. However, our data indicate that social disconnectedness and stress account for a larger proportion of cases than mood disorders. Should these associations prove to be causal, then interventions that succeeded in addressing these issues would contribute the most to reducing suicidal ideation and, possibly, suicidal behaviour in later life.

\section{Declaration of interest}

None.
Thoughts about death are not uncommon in later life, particularly in extreme old age. ${ }^{1}$ Their presence is strongly associated with symptoms of depression and anxiety, ${ }^{1}$ and in some cases may evolve to self-harm ideation and suicide. ${ }^{2,3}$ Various factors are thought to contribute to the development of self-harm ideation in later life, the most common being the presence of clinically significant depressive symptoms. ${ }^{4}$ A review of psychological autopsy studies completed in the past decade showed that $46-86 \%$ of older adults who died by suicide displayed symptoms consistent with a mood disorder in the weeks preceding their death, ${ }^{5}$ which suggests that the successful prevention and management of depression might reduce the rate of suicide in this age group, particularly among men. ${ }^{6}$ Not surprisingly, most suicide prevention strategies introduced to date have focused on increasing the detection of depression and optimising its management in primary care. ${ }^{7,8}$ Collaborative care strategies, for example, have been successful at reducing the severity of depressive symptoms and the prevalence of suicide ideation in older adults with depression, ${ }^{9}$ but benefits have been modest and the long-term sustainability of such programmes remains uncertain. $^{10}$

One possible explanation of the limited success of depressionfocused interventions in reducing the prevalence of suicide ideation in later life is that other, untargeted factors may drive this association. For example, the rate of suicide in later life is higher in men than in women, as well as in people with pain and multiple medical morbidities, ${ }^{11}$ marked feelings of hopelessness, ${ }^{12}$ financial concerns and loneliness. ${ }^{13}$ There is also evidence that older people with overlapping anxiety and depressive symptoms may be particularly vulnerable to the onset of suicide ideation, ${ }^{14}$ and it is possible that the increasing sense of edginess and psychological discomfort associated with anxiety are important contributing factors that demand attention and treatment. However, given the multitude of factors that have been associated with suicide ideation and behaviour in later life, the design of data-driven preventive strategies would benefit from data derived from large community studies that measure simultaneously the relevant demographic, lifestyle, psychosocial and clinical variables in order to minimise confounding and enhance the generalisability of the data. We report data derived from a community study of over 20000 older Australians, intended to determine the independent contribution of demographic, lifestyle, socioeconomic, psychiatric and medical factors to the presence of suicide ideation.

\section{Method}

\section{Study design and participants}

Our analyses are based on data originating from the Depression and Early Prevention of Suicide in General Practice study. Details regarding the recruitment have been reported elsewhere. ${ }^{15}$ Briefly, between May and December 2005 all patients aged 60 years or over of participating primary care practices in New South Wales, Victoria, Queensland, South Australia and Western Australia were sent a questionnaire, a personalised cover letter from their general practitioner, project information, a consent form and a reply-paid envelope addressed to the project office. We asked potential participants to return the questionnaires (blank in the case of 
non-consent) so that we could estimate the true denominator of the target population. We received 22258 questionnaires with written informed consent. Another 9087 questionnaires were returned not completed, 2934 were returned to the sender because the person named on the envelope was not known at the address and 820 failed to be posted because of mishandling of the envelopes by the company organising the mail-out (the total number of questionnaires tracked was 35099 ). A small number of people who consented were found to be ineligible because they were under 60 years old $(n=120)$ or did not reside in the community (nursing home; $n=54$ ). A further 229 questionnaires had incomplete data on age and 30 were duplicates, leaving a sample of 21825 persons, of whom 21290 reported information on suicide ideation. The ethics committees of the University of Western Australia, the University of Melbourne and the Royal Australian College of General Practitioners approved the study protocol and all participants provided informed consent.

\section{Primary outcome}

The outcome of interest was suicidal ideation according to the Depressive Symptom Inventory - Suicidality Subscale. ${ }^{16,17}$ This is a four-item self-report questionnaire that assesses 2-weekly suicidal ideation (thoughts about killing oneself), degree to which a plan has been formulated, ability to control suicidal thoughts (from complete to little or no control) and suicidal impulses (intensity of impulse to act on suicidal thoughts). Scores on each item range from 0 to 3 , with higher scores reflecting greater severity of suicidal ideation. Older adults who scored 1 or more on any of these four questions were considered to represent prevalent cases of suicidal ideation.

\section{Other study measures}

Participants provided information about their gender, place of birth (Australia v. overseas), marital status, highest educational achievement and date of birth, which we used to calculate their age (in years). We also asked them whether, as a rule, they took at least half an hour of moderate or vigorous exercise on five or more days of the week (yes/no), ${ }^{18}$ whether they smoked (never, past, current) and how many standard drinks they normally had on each typical day of the week. People who reported consuming seven or more drinks on any one day or who had three or more drinks nearly every day were considered risk drinkers. ${ }^{19}$ We used self-reported height and weight to calculate the body mass index (BMI), and classified as overweight or obese participants with a BMI of $25 \mathrm{~kg} / \mathrm{m}^{2}$ or above. We collected information about living arrangements (alone $v$. with others) and used the Duke Social Support Index to measure perceived social support. ${ }^{20}$ Scores on this index of 26.5 or below represented the lowest quartile of scores in our sample and were used as an indicator of poor social support. Respondents scoring in the lowest quintile of the Index of Relative Socio-Economic Disadvantage were considered socially disadvantaged. ${ }^{21}$ In addition, participants indicated whether they attended religious services regularly (yes/no) and whether they were experiencing financial strain (distinctly or very much $v$. slightly or not at all). Study participants recorded whether they had lost a parent or experienced physical or sexual abuse before they were 15 years old (yes/no). They also indicated whether any immediate family member had died by suicide (yes/no).

Older adults in the study recorded whether a doctor had ever told them they had an anxiety disorder or depression (yes/ no) and whether they had ever attempted to kill themselves in their lifetime (never/once or more than once) or during the past year (never/once or more than once). Participants rated their overall health as fair/poor or good to excellent, and indicated whether pain had interfered with their usual activities during the preceding 4 weeks (no/little or moderately to extremely). We assessed prevalent symptoms of anxiety and depression with the Hospital Anxiety and Depression Scale (HADS). ${ }^{22}$ Possible scores range from 0 to 21 for each subscale (HADS-A for anxiety and HADS-D for depression), with scores of 11 or above indicating the presence of clinically significant anxiety or depression. ${ }^{22}$ We used the scores on these subscales to assign participants to one of four groups: no anxiety or depression (HADS-A and HADS-D <11); anxiety but no depression (HADS-A $\geqslant 11$ and HADS-D <11); depression but no anxiety (HADS-D $\geqslant 11$ and HADS-A $<11$ ); and anxiety and depression (HADS-A $\geqslant 11$ and HADS-D $\geqslant 11$ ). Participants recorded whether they had ever been told by a doctor that they had any of the following conditions (yes/no): arthritis, diabetes, hypertension, stroke, heart attack or angina, heart failure, poor circulation in the legs, asthma, emphysema, osteoporosis, cancer (except skin cancer), dementia, thyroid disease and head injury. We grouped them according to the number of reported medical morbidities: $0 ; 1$ or $2 ; 3$ or $4 ; 5$ or more. Finally, we asked participants to record the names of any medicines they had used during the preceding 2 weeks, and from this list we retrieved information about the use of benzodiazepines or antidepressants (any class).

\section{Statistical analysis}

Data were managed and analysed with Stata version 12.0 for Mac. We used descriptive statistics to summarise the data and crosstabulation to determine their distribution according to the presence of suicide ideation. We used Pearson's chi-squared statistic to estimate whether differences in the distribution of measured factors between the groups could have arisen by chance. We then investigated the association between measured factors (independent variables) and the suicide ideation grouping (dependent variable) using logistic regression. The reported odds ratio and its $95 \%$ confidence interval were derived from a multivariate model that included all measured factors. We subsequently created a parsimonious model of suicidal ideation where only variables associated at a significance level of $P<0.05$ were retained (stepwise backwards regression), and used the effect estimates to calculate the fraction of suicidal ideation that could be attributed to the exposures in the model (command: punaf). In this study the population attributable fraction (PAF) offers an indication of the proportion of people with suicidal ideation who would no longer report suicidal thoughts if the relevant exposure (e.g. pain) could be removed (all other variables in the model held constant). This approach assumes that the exposure is causally related to the outcome. Alpha was set at 5\% and all probability tests used were two-tailed.

\section{Results}

Of 21290 participants, 1023 (4.8\%) acknowledged the presence of suicide ideation during the preceding 2 weeks. The age range of the sample was 60-101 years, and the mean age of participants with suicide ideation was 70.5 (s.d. $=7.9$ ) years compared with 71.9 (s.d. $=7.6)$ years for those without such ideation $(t=5.38$, $P<0.001)$. Table 1 shows the distribution of demographic, lifestyle and socioeconomic characteristics of those with and without suicide ideation, and Table 2 shows participants' clinical characteristics. There was a significant excess of men, migrants, unmarried and highly educated people among the ideation group, who were also more physically inactive, overweight or obese, past 


\begin{tabular}{|c|c|c|c|}
\hline & $\begin{array}{l}\text { No suicidal ideation } \\
(n=20267), n(\%)\end{array}$ & $\begin{array}{l}\text { Suicidal ideation } \\
(n=1023), n(\%)\end{array}$ & $\begin{array}{l}\text { Univariate odds ratio of suicidal } \\
\text { ideation }(95 \% \mathrm{Cl})\end{array}$ \\
\hline \multicolumn{4}{|l|}{ Demographic information } \\
\hline \multicolumn{4}{|l|}{ Age, years } \\
\hline $60-64$ & $4117(20.3)$ & $311(30.4)$ & 1 (reference) \\
\hline $65-69$ & $4831(23.8)$ & $230(22.5)$ & $0.6(0.5-0.8)$ \\
\hline 70-74 & $4144(20.4)$ & $190(18.6)$ & $0.6(0.5-0.7)$ \\
\hline 75-79 & 3494 (17.2) & $134(13.1)$ & $0.5(0.4-0.6)$ \\
\hline $80-84$ & $2355(11.6)$ & $87(8.5)$ & $0.5(0.4-0.6)$ \\
\hline $85+$ & $1326(6.5)$ & $71(6.9)$ & $0.7(0.5-0.9)$ \\
\hline Male gender & $8337(41.2)$ & $463(45.3)$ & $1.2(1.0-1.3)$ \\
\hline Migrant & $5157(25.5)$ & $296(29.0)$ & $1.2(1.0-1.4)$ \\
\hline Not married & $6552(32.4)$ & $465(45.6)$ & $1.8(1.5-2.0)$ \\
\hline Higher education & $2930(14.7)$ & $184(18.4)$ & $1.3(1.1-1.5)$ \\
\hline Had children & $18486(97.0)$ & $909(97.0)$ & $1.0(0.7-1.5)$ \\
\hline \multicolumn{4}{|l|}{ Lifestyle variables } \\
\hline Physically inactive & 7321 (36.5) & $492(48.4)$ & $1.6(1.4-1.9)$ \\
\hline Overweight or obese & $11544(64.1)$ & $623(69.4)$ & $1.3(1.1-1.5)$ \\
\hline \multicolumn{4}{|l|}{ Smoking } \\
\hline Never & $10565(52.4)$ & $407(40.2)$ & 1 (reference) \\
\hline Past & $8399(41.7)$ & $458(45.2)$ & $1.4(1.2-1.6)$ \\
\hline Current & $1197(5.9)$ & $148(14.6)$ & $3.2(2.6-3.9)$ \\
\hline \multicolumn{4}{|l|}{ Alcohol use } \\
\hline None & $4956(24.7)$ & $270(26.7)$ & 1 (reference) \\
\hline Non-risk use & $12611(62.9)$ & $578(57.2)$ & $0.8(0.7-1.0)$ \\
\hline Risky use & $2490(12.4)$ & $162(16.0)$ & $1.2(1.0-1.5)$ \\
\hline \multicolumn{4}{|l|}{ Socioeconomic history } \\
\hline Living alone & $4797(23.7)$ & $321(31.5)$ & $1.5(1.3-1.7)$ \\
\hline Poor social support & 4419 (22.5) & 665 (67.4) & $7.1(6.2-8.2)$ \\
\hline Social disadvantage & $3905(19.9)$ & $189(19.1)$ & $1.0(0.8-1.1)$ \\
\hline No religious practice & $10865(53.8)$ & $638(62.5)$ & $1.4(1.3-1.6)$ \\
\hline Financial strain & $1873(9.6)$ & $285(29.0)$ & $3.9(3.3-4.5)$ \\
\hline Loss of a parent during childhood & $2544(12.6)$ & $171(16.8)$ & $1.4(1.2-1.7)$ \\
\hline Childhood physical abuse & $1218(6.0)$ & $194(19.1)$ & $3.7(3.1-4.3)$ \\
\hline Childhood sexual abuse & $1221(6.0)$ & $165(16.2)$ & $3.0(2.6-3.6)$ \\
\hline Suicide in family & $1137(5.6)$ & $117(11.6)$ & $2.2(1.8-2.7)$ \\
\hline
\end{tabular}

Table 2 Clinical characteristics of the participants categorised by suicidal ideation

\begin{tabular}{|lccc|}
\hline & $\begin{array}{c}\text { No suicidal ideation } \\
(n=20267), n(\%)\end{array}$ & $\begin{array}{c}\text { Suicidal ideation } \\
(n=1023), n(\%)\end{array}$ & $\begin{array}{c}\text { Univariate odds ratio of suicidal } \\
\text { ideation (95\% Cl) }\end{array}$ \\
\hline Past anxiety disorder & $1696(8.4)$ & $269(26.3)$ & $3.9(3.4-4.2)$ \\
\hline Past depressive disorder & $3149(15.5)$ & $566(55.3)$ & $6.7(5.9-7.7)$ \\
\hline HADS status & $17943(93.6)$ & $509(52.1)$ & 1 (reference) \\
Neutral & $698(3.6)$ & $173(17.7)$ & $8.7(7.3-10.5)$ \\
Anxiety & $291(1.5)$ & $92(9.4)$ & $11.1(8.7-14.3)$ \\
Depression & $244(1.3)$ & $203(20.8)$ & $29.3(23.9-36.0)$ \\
Anxiety and depression & $368(1.8)$ & $131(12.8)$ & $7.9(6.4-9.8)$ \\
\hline Past suicide attempt & $15(0.1)$ & $25(2.4)$ & $33.8(17.8-64.3)$ \\
\hline Suicide attempt past year & $5883(29.2)$ & $595(58.6)$ & $3.4(3.0-3.9)$ \\
\hline Pain & $4222(21.0)$ & $523(51.3)$ & $4.0(3.5-4.5)$ \\
\hline Poor perceived health & & & 1 (reference) \\
\hline Medical morbidities & $2004(9.9)$ & $63(6.2)$ & $1.2(0.9-1.6)$ \\
\hline 1-2 & $9765(48.2)$ & $370(36.2)$ & $1.9(1.5-2.5)$ \\
\hline 3-4 & $6398(31.6)$ & $383(37.4)$ & $3.1(2.3-4.2)$ \\
\hline 5+ & $2100(10.4)$ & $207(20.2)$ & $2.7(2.3-3.2)$ \\
\hline Use of benzodiazepines & $1404(6.9)$ & $173(16.9)$ & $3.9(3.4-4.5)$ \\
\hline Use of antidepressants & $2202(10.9)$ & $332(32.4)$ & \\
\hline HADS, Hospital Anxiety and Depression Scale. & & & \\
\hline
\end{tabular}

or current smokers and risky alcohol users. Compared with those who denied ideation, a greater proportion of people experiencing thoughts of suicide lived alone, had poor social support, did not practise a religion, were experiencing financial stress, had lost a parent or had been recipients of physical or sexual abuse during childhood, and reported a history of suicide in the family. More people in the suicide ideation group than in the non-ideation group had a history of anxiety or depressive disorder, a past suicide attempt, pain or poor self-perceived health and multiple medical morbidities. The proportion of respondents with clinically 
significant anxiety, depression or comorbid anxiety and depression was larger in the suicide ideation group. Finally, more people reporting suicidal ideation were using benzodiazepines and antidepressants.

We used multivariate logistic regression to determine the odds of suicide ideation for all measured factors listed in Tables 1 and 2 (all factors forced simultaneously into the model). The results are illustrated in Fig. 1. Male gender, possessing a higher degree, being a current smoker, living alone, being in the lowest quintile of social support, practising no religion, experiencing financial stress, reporting a history of suicide in the family or of past depression, presenting clinically significant symptoms of anxiety, depression or of comorbid anxiety and depression, experiencing moderate to severe pain or poor self-perceived health, and using antidepressants were all independently associated with increased risk of prevalent suicide ideation (pseudo $R^{2}=25.5 \%$ for the multivariate model).
The parsimonious logistic regression model of suicide ideation, which included only exposures associated at $P<0.05$, appears in Table 3 (pseudo $R^{2}=25.4 \%$ ). Variables significantly associated with suicide ideation were male gender, higher education, current smoking, living alone, poor social support, no religious practice, financial strain, childhood physical abuse, history of suicide in the family, past depression, current anxiety, depression or comorbid anxiety and depression, past suicide attempt, pain, poor self-perceived health and current use of antidepressants. We also calculated the PAF for each of the independent variables of the parsimonious model (Table 3). Poor social support was associated with a PAF of $38.0 \%$, followed by history of depression (23.6\%), concurrent anxiety and depression (19.7\%), prevalent anxiety (15.1\%), pain (13.7\%) and no religious practice $(11.4 \%)$. All other variables in the model were associated with significant PAFs that were lower than $10 \%$.

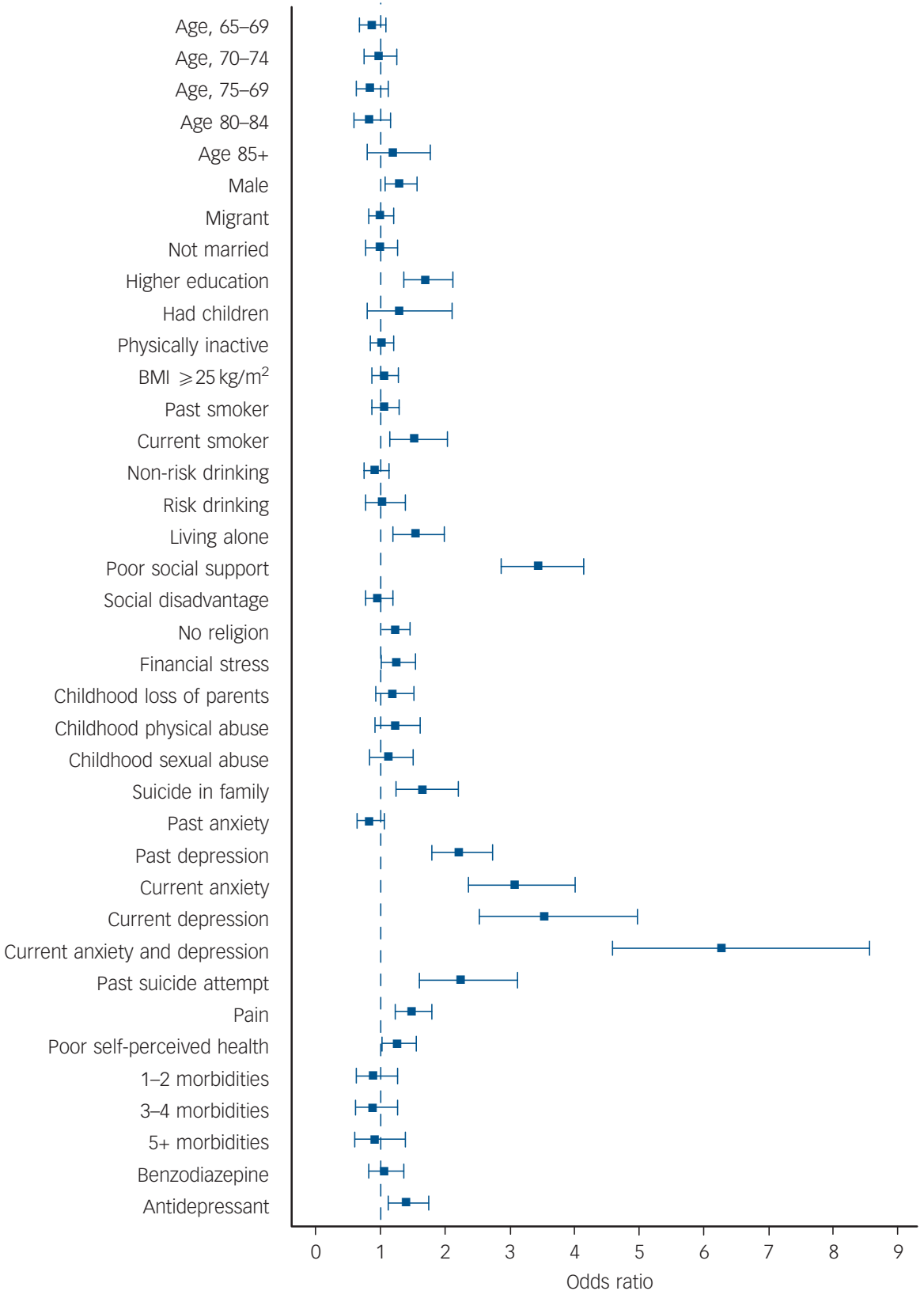

Fig. 1 Odds ratio of suicide ideation according to various demographic, lifestyle, psychosocial and clinical factors 
Table 3 Odds ratio and population attributable fraction of suicide ideation according to exposures derived from a multivariate logistic regression model

\begin{tabular}{|lcc|}
\hline & OR (95\% CI) & PAF\% (95\% CI) \\
\hline Male gender & $1.3(1.1-1.5)$ & $7.2(1.9-12.3)$ \\
\hline Higher education & $1.7(1.4-2.1)$ & $6.6(3.8-9.2)$ \\
\hline Current smoker $(v$. never smoked) & $1.3(1.0-1.7)$ & $4.7(0.3-8.9)$ \\
\hline Living alone & $1.5(1.3-1.8)$ & $8.1(4.4-11.7)$ \\
\hline Poor social support & $3.1(2.6-3.7)$ & $38.0(32.2-43.4)$ \\
\hline No religious practice & $1.3(1.1-1.5)$ & $11.4(4.3-18.0)$ \\
\hline Financial strain & $1.3(1.1-1.6)$ & $4.6(1.2-8.0)$ \\
\hline Childhood physical abuse & $1.3(1.1-1.6)$ & $3.0(0.3-5.6)$ \\
\hline Suicide in family & $1.5(1.2-2.0)$ & $2.9(0.9-4.8)$ \\
\hline Past depressive disorder & $2.3(1.9-2.8)$ & $23.6(17.9-28.9)$ \\
\hline HADS status & $3.3(2.6-4.1)$ & $15.1(11.3-18.7)$ \\
\hline Anxiety & $3.6(2.7-5.0)$ & $8.8(6.0-11.6)$ \\
\hline Depression & $7.1(5.4-9.3)$ & $19.7(16.5-22.8)$ \\
\hline Anxiety and depression & $2.3(1.7-3.1)$ & $4.5(2.7-6.3)$ \\
\hline Past suicide attempt & $1.5(1.2-1.7)$ & $13.7(7.3-19.6)$ \\
\hline Pain & $1.3(1.1-1.5)$ & $7.6(1.7-13.2)$ \\
\hline Poor perceived health & $1.3(1.0-1.6)$ & $4.8(0.7-8.7)$ \\
\hline Antidepressant use & & \\
\hline HADs, Hospital Anxiety and Depression Scale; PAF, population attributable fraction. \\
\hline
\end{tabular}

\section{Discussion}

The results of this large community-based study show that nearly $5 \%$ of older Australians acknowledged suicidal thoughts in the preceding 2 weeks, a rate that is within the range of suicide ideation in the preceding month $(2.3-15.9 \%)$ noted in world literature. $^{23}$ Our study was sufficiently large to explore the independent association with suicide ideation of numerous demographic, lifestyle, socioeconomic and clinical variables, including male gender, higher education, smoking, living alone and having limited social support, not practising a religion, experiencing financial stress, family history of suicide, history of depression or of suicide attempt, current anxiety, depression or comorbid anxiety and depression, pain, poor self-perceived health and use of an antidepressant. The risk of suicide ideation was particularly high among people with concurrent anxiety and depression, followed by those with prevalent depression but no anxiety and by those with prevalent anxiety but no depression. Poor social support was associated with the largest PAF, followed by a self-reported history of medically diagnosed depression, prevalent concurrent anxiety and depression, prevalent anxiety with no depression, pain and no religious practice.

\section{Limitations of the study}

Before discussing the implications of our findings we should consider how the characteristics of the study could potentially affect the interpretation of the results. We recruited a large sample of community-dwelling older adults that was broadly representative of the Australian community, ${ }^{24}$ although the response rate to invitations was not optimal and may have introduced bias as a result of healthy people being more willing to participate. The consequence of such a bias would have been a reduction in the number of people who were unwell, possibly including cases of suicide ideation, anxiety and depression. This might have reduced the strength of the association between suicide ideation and health morbidities, as well as our ability to adjust the statistical models for poor health. Hence, the effect estimates of the association between the exposures and suicide ideation may have decreased because of selection bias or increased because of confounding. Our study was sufficiently large to declare as significant odds ratios as small as 1.3, and as odds ratios smaller than this are unlikely to be of clinical significance, it seems improbable that our results would have been severely affected by loss of power. In addition, the effect of the number of medical morbidities in the analysis was not significant, which suggests that confounding due to poor health should not have influenced the results substantially.

We used a well-validated approach to assess suicide ideation, ${ }^{16,17}$ but acknowledge that thinking about killing oneself is not the same as attempting or completing suicide. Thus, it may be argued that our findings are relevant for suicidal thoughts rather than for behaviour. Indeed, suicide attempt and completion are rare occurrences compared with suicidal ideation in later life, ${ }^{2,4}$ and most people who consider ending their lives never attempt suicide. However, suicidal thoughts are nearly always present before suicide completion, ${ }^{25,26}$ so that reducing the prevalence of suicide ideation is a necessary step of suicide prevention programmes.

The interpretation of our results should also take into account the underlying assumption that the association between the exposures (risk factors) and suicide ideation is causal. Causality cannot be inferred from cross-sectional studies such as ours, nor can we dismiss the possibility of reverse associations entirely. For example, it is conceivable that people who think about suicide feel distressed by such a thought, and this in turn could lead to the onset of clinically significant symptoms of anxiety. In this scenario, anxiety would be a consequence rather than a cause of suicidal thoughts. However, evidence from other sources suggests that anxiety precedes the onset of suicide ideation, ${ }^{27}$ thereby undermining the hypothesis of reverse causality. The remission of most suicidal thoughts associated with the successful treatment of depression would similarly argue against the possibility that such ideas would cause depressive symptoms. ${ }^{28}$ Finally, other factors associated with prevalent suicide ideation in our study would seem less susceptible to reverse causality (e.g. male gender, higher education, smoking, poor social support, no religious practice, childhood physical abuse, history of suicide in the family, past suicide attempt, past depressive episode), but uncertainty remains for some of the exposures that we measured, such as use of antidepressants and subjective experience of pain.

We had access to information about relevant exposures, but acknowledge that using the HADS to establish the presence of anxiety and depression is not the same as using structured clinical interviews or diagnostic criteria. ${ }^{22,29}$ Hence, we cannot be certain that our findings would be directly transferable to patients with anxiety and mood disorders defined according to DSM criteria. Finally, we used a reliable measure of perceived social support, ${ }^{20}$ but did not have access to objective information about social network structure and size, actual support received or disability. ${ }^{30}$ Therefore, it seems possible that some of the associations that we observed could be explained, at least in part, by unmeasured factors.

\section{Interpretation}

The results of this study indicate that the risk of suicidal ideation is 7 times as high in older adults with concurrent anxiety and depression as in those without anxiety or depression, which suggests that everyone in this age group with such a clinical 
presentation should be screened for the presence of suicidal thoughts. ${ }^{1,31}$ Similarly, in this sample the odds of suicide ideation were respectively 3.6 and 3.3 times higher among those with prevalent depression but no anxiety and those with prevalent anxiety but no depression. ${ }^{2,27,32}$ Although perceived poor social support increased the odds of suicide ideation by 3.1 times, it was associated with the largest PAF $(38.0 \%)$ in our model, followed by self-reported history of depression (PAF 23.6\%), prevalent concurrent anxiety and depression (PAF 19.7\%), prevalent anxiety but no depression (PAF 15.1\%), pain (PAF $13.7 \%$ ) and no religious practice (PAF 11.4\%). Notwithstanding the statistical robustness of the PAFs associated with measured exposures in our study, some caution is required when interpreting these results. A key assumption underlying valid estimates is that the relationship between the exposures and the outcome is causal and independent. ${ }^{33}$ We have modelled our data to ensure the reported PAFs were independent, but are unable to establish causality from our cross-sectional study; such a shortcoming can only be overcome by properly conducted randomised trials. Other major contributing factors included male gender, higher education, smoking, living alone, no religious practice, financial strain, childhood physical abuse, family history of suicide, past suicide attempt, poor perceived health and antidepressant use, the importance of which has been previously highlighted by others. ${ }^{4,5,34,35}$ However, we are not aware of any study that had sufficient power to examine the independent contributions of the wide range of demographic, lifestyle, socioeconomic and clinical factors that we examined in this study.

Our findings suggest that suicide prevention strategies limited to older adults with mood disorders, although efficacious, ${ }^{28,36}$ may have only limited impact on the overall prevalence of suicidal thoughts in the population. Indicated or selective preventive programmes, targeting people with symptoms or those at risk, might have greater impact on the prevalence of suicidal ideation if relevant social, demographic, lifestyle and medical factors were also addressed. In fact, our data indicate that social factors (poor perceived social support and living alone) may have a particularly prominent role in this age group and, although their management is challenging, preliminary evidence suggests that interventions such as telephone support and befriending might be feasible and helpful. ${ }^{37}$ In fact, one might speculate that women respond better than men to suicide prevention programmes because their participation reduces the sense of social isolation associated with living alone, a factor that may be less relevant to men. ${ }^{35}$ Nonetheless, increasing opportunities for social interaction may fail to reduce the experience of loneliness and social isolation of people with mental health disorders if maladaptive social cognitions (e.g. heightened sensitivity to perceived social threats) are not managed appropriately. ${ }^{38}$ Indeed, a detailed meta-analysis of trials designed to reduce loneliness concluded that interventions that addressed maladaptive social cognitions were the most helpful. ${ }^{39}$ If the findings reported for loneliness are equally applicable to perceived social support in later life, future preventive programmes will need to consider whether to increase opportunities for social interaction, target maladaptive social cognitions, or both, in order to decrease the prevalence of suicidal ideation. Our results also support the view that, consistent with models of late-life depression, ${ }^{40}$ a life-cycle approach to suicide prevention must be considered, as early and midlife factors may contribute to modulate the presence of late psychosocial risk factors and clinical presentations. Well-designed randomised trials targeting vulnerable populations are now needed to guide policy and practice.
Osvaldo P. Almeida, MD, PhD, FRANZCP, School of Psychiatry and Clinica Neurosciences, and Western Australian Centre for Health and Ageing, Centre for Medical Research, University of Western Australia, and Department of Psychiatry, Royal Perth Hospital, Perth; Brian Draper, MBBS, MD, FRANZCP, School of Psychiatry, University of New South Wales, Randwick; John Snowdon, MBBS, MD, FRANZP, Discipline of Psychiatry, Sydney Medical School, University of Sydney; Nicola T. Lautenschlager, MD, FRANZCP, School of Psychiatry and Clinical Neurosciences and Western Australian Centre for Health and Ageing, Centre for Medical Research, University of Western Australia, Perth, and Academic Unit for Psychiatry of Old Age, St Vincent's Health, Department of Psychiatry, University of Melbourne; Jane Pirkis, BA, MPsych, MAppEpid, PhD, School of Population Health, University of Melbourne; Gerard Byrne, MBBS, PhD, FRANZCP, School of Medicine, University of Queensland, Herston; Moira Sim, MBBS, FRACGP, School of Medical Sciences, Edith Cowan University, Perth; Nigel Stocks, BSC, MBBS, MD, DipPH, FRACGP, Discipline of General Practice, University of Adelaide; Leon Flicker, MBBS, PhD, FRACP, Western Australian Centre for Health and Ageing, Centre for Medical Research and School of Medicine and Pharmacology, University of Western Australia, and Department of Geriatric Medicine, Royal Perth Hospital, Perth; Jon J. Pfaff, BA, MSC, PhD, Schoo of Psychiatry and Clinical Neurosciences and Western Australian Centre for Health and Ageing, Centre for Medical Research, University of Western Australia, Perth, Australia

Correspondence: Professor Osvaldo P. Almeida, Western Australia Centre for Health and Ageing (M573), University of Western Australia, 35 Stirling Highway, Crawley, Perth, WA 6009, Australia. Email: osvaldo.almeida@uwa.edu.au

First received 6 Feb 2012, final revision 2 Aug 2012, accepted 10 Oct 2012

\section{Funding}

The study was supported by the project grant number 353569 from the National Health and Medical Research Council of Australia and by an infrastructure grant from beyondblue Australia.

\section{Acknowledgements}

The investigators thank participants and research staff for their generous contribution

\section{References}

1 Bartels SJ, Coakley E, Oxman TE, Constantino G, Oslin D, Chen $\mathrm{H}$, et al. Suicidal and death ideation in older primary care patients with depression, anxiety, and at-risk alcohol use. Am J Geriatr Psychiatry 2002; 10: 417-27.

2 Lawrence D, Almeida OP, Hulse GK, Jablensky AV, Holman CD. Suicide and attempted suicide among older adults in Western Australia. Psychol Med 2000; 30: 813-21.

3 Pfaff JJ, Almeida OP. Identifying suicidal ideation among older adults in a general practice setting. J Affect Disord 2004; 83: 73-7.

4 Pfaff JJ, Almeida OP. Detecting suicidal ideation in older patients: identifying risk factors within the general practice setting. Br J Gen Pract 2005; 55: 269-73.

5 Conwell Y, Van Orden K, Caine ED. Suicide in older adults. Psychiatr Clin North Am 2011; 34: 451-68, ix.

6 Mann JJ, Apter A, Bertolote J, Beautrais A, Currier D, Haas A, et al. Suicide prevention strategies: a systematic review. JAMA 2005; 294: 2064-74.

7 Bruce ML, Pearson JL. Designing an intervention to prevent suicide: PROSPECT (Prevention of Suicide in Primary Care Elderly: Collaborative Trial). Dialogues Clin Neurosci 1999; 1: 100-12.

8 Rihmer Z, Rutz W, Pihlgren H. Depression and suicide on Gotland. An intensive study of all suicides before and after a depression-training programme for general practitioners. J Affect Disord 1995; 35: 147-52.

9 Bruce ML, Ten Have TR, Reynolds CF, Katz II, Schulberg HC, Mulsant BH, et al. Reducing suicidal ideation and depressive symptoms in depressed older primary care patients: a randomized controlled trial. JAMA 2004; 291 1081-91.

10 Gilbody S, Bower P, Whitty P. Costs and consequences of enhanced primary care for depression. Systematic review of randomised economic evaluations. Br J Psychiatry 2006; 189: 297-308.

11 Van Orden K, Conwell Y. Suicides in late life. Curr Psychiatry Rep 2011; 13: 234-41.

12 Rifai AH, George CJ, Stack JA, Mann JJ, Reynolds CF. Hopelessness in suicide attempters after acute treatment of major depression in late life. Am J Psychiatry 1994; 151: 1687-90.

13 Rubenowitz E, Waern $\mathrm{M}$, Wilhelmson $\mathrm{K}$, Allebeck P. Life events and psychosocial factors in elderly suicides - a case-control study. Psychol Med 2001; 31: 1193-202. 
14 Mykletun A, Bjerkeset O, Dewey M, Prince M, Overland S, Stewart R. Anxiety, depression, and cause-specific mortality: the HUNT study. Psychosom Med 2007; 69: 323-31.

15 Almeida OP, Pirkis J, Kerse N, Sim M, Flicker L, Snowdon J, et al. A randomized trial to reduce the prevalence of depression and self-harm behavior in older primary care patients. Ann Fam Med 2012; 10: 347-56.

16 Metalsky Gl, Joiner TE. The hopelessness depression symptom questionnaire. Cog Therap Res 1997; 21: 359-84.

17 Joiner $T E$, Rudd MD. Disentangling the interrelations between hopelessness, Ioneliness, and suicidal ideation. Suicide Life Threat Behav 1996; 26: 19-26.

18 Elley CR, Kerse NM, Arroll B. Why target sedentary adults in primary health care? Baseline results from the Waikato Heart, Health, and Activity Study. Prev Med 2003; 37: 342-8

19 National Health and Medical Research Council. Australian Guidelines to Reduce Health Risks From Drinking Alcohol. Commonwealth of Australia, 2009

20 Koenig HG, Westlund RE, George LK, Hughes DC, Blazer DG, Hybels C. Abbreviating the Duke Social Support Index for use in chronically ill elderly individuals. Psychosomatics 1993; 34: 61-9.

21 Pink B. An Introduction to Socio-Economic Indexes for Areas (SEIFA) 2006. Australian Bureau of Statistics, 2008.

22 Zigmond AS, Snaith RP. The hospital anxiety and depression scale. Acta Psychiatr Scand 1983; 67: 361-70.

23 De Leo D, Krysinska K, Bertolote JM, Fleischmann A, Wasserman D. Suicidal behaviours on the five continents among the elderly. In Oxford Textbook of Suicidology and Suicide Prevention (eds D Wasserman, C Wasserman): 693-702. Oxford University Press, 2009.

24 Pirkis J, Pfaff J, Williamson M, Tyson O, Stocks N, Goldney R, et al. The community prevalence of depression in older Australians. J Affect Disord 2009; 115: 54-61.

25 Waern M, Beskow J, Runeson B, Skoog I. Suicidal feelings in the last year of life in elderly people who commit suicide. Lancet 1999; 354: 917-8.

26 Conwell $Y$, Duberstein PR, Cox C, Herrmann J, Forbes N, Caine ED. Age differences in behaviors leading to completed suicide. Am J Geriatr Psychiatry 1998; 6: 122-6.

27 Sareen J, Cox BJ, Afifi TO, de Graaf R, Asmundson GJ, ten Have M, et al. Anxiety disorders and risk for suicidal ideation and suicide attempts: a population-based longitudinal study of adults. Arch Gen Psychiatry 2005; 62 1249-57.

28 Alexopoulos GS, Reynolds CF, Bruce ML, Katz IR, Raue PJ, Mulsant BH, et al. Reducing suicidal ideation and depression in older primary care patients: 24-month outcomes of the PROSPECT study. Am J Psychiatry 2009; 166 $882-90$

29 Bjelland I, Dahl AA, Haug TT, Neckelmann D. The validity of the Hospital Anxiety and Depression Scale. An updated literature review. J Psychosom Res 2002; 52: 69-77.

30 Dennis M, Baillon S, Brugha T, Lindesay J, Stewart R, Meltzer $H$. The influence of limitation in activity of daily living and physical health on suicidal ideation: results from a population survey of Great Britain. Soc Psychiatry Psychiatr Epidemiol 2009; 44: 608-13.

31 Pfeiffer PN, Ganoczy D, Ilgen M, Zivin K, Valenstein M. Comorbid anxiety as a suicide risk factor among depressed veterans. Depress Anxiety 2009; 26 : 752-7.

32 Bolton JM, Robinson J. Population-attributable fractions of Axis I and Axis II mental disorders for suicide attempts: findings from a representative sample of the adult, noninstitutionalized US population. Am J Public Health 2010; 100: $2473-80$

33 Rockhill B, Newman B, Weinberg C. Use and misuse of population attributable fractions. Am J Public Health 1998; 88: 15-9.

34 Turvey $\mathrm{CL}$, Conwell $\mathrm{Y}$, Jones MP, Phillips $\mathrm{C}$, simonsick $\mathrm{E}$, Pearson $\mathrm{J}$, et al. Risk factors for late-life suicide: a prospective, community-based study. Am J Geriatr Psychiatry 2002; 10: 398-406.

35 Lapierre S, Erlangsen A, Waern M, De Leo D, Oyama H, Scocco P, et al. A systematic review of elderly suicide prevention programs. Crisis 2011; 32 : 88-98.

36 Unutzer J, Tang L, Oishi S, Katon W, Williams JW, Hunkeler E, et al. Reducing suicidal ideation in depressed older primary care patients. J Am Geriatr SoC 2006; 54: 1550-6.

37 De Leo D, Dello Buono M, Dwyer J. Suicide among the elderly: the long-term impact of a telephone support and assessment intervention in northern Italy. Br J Psychiatry 2002; 181: 226-9.

38 Meltzer $\mathrm{H}$, Bebbington P, Dennis MS, Jenkins R, McManus S, Brugha TS Feelings of loneliness among adults with mental disorder. Soc Psychiatry Psychiatr Epidemiol 2012; May 9, doi: 10.1007/S00127-012-0515-8 (epub ahead of print).

39 Masi CM, Chen HY, Hawkley LC, Cacioppo JT. A meta-analysis of interventions to reduce loneliness. Pers Soc Psychol Rev 2011; 15: 219-66.

40 Almeida OP, Alfonso H, Pirkis J, Kerse N, Sim M, Flicker L, et al. A practical approach to assess depression risk and to guide risk reduction strategies in later life. Int Psychogeriatr 2011; 23: 280-91. 